This item was submitted to Loughborough's Research Repository by the author.

Items in Figshare are protected by copyright, with all rights reserved, unless otherwise indicated.

\title{
Bound states in coupled guides. I. Two dimensions.
}

PLEASE CITE THE PUBLISHED VERSION

LICENCE

CC BY-NC-ND 4.0

\section{REPOSITORY RECORD}

Linton, C.M., and Keith Ratcliffe. 2019. "Bound States in Coupled Guides. I. Two Dimensions.". figshare. https://hdl.handle.net/2134/307. 


\title{
Bound states in coupled guides. I. Two dimensions.
}

\section{M. Linton and K. Ratcliffe ${ }^{1}$}

\begin{abstract}
Bound states that can occur in coupled quantum wires are investigated. We consider a two-dimensional configuration in which two parallel waveguides (of different widths) are coupled laterally through a finite length window and construct modes which exist local to the window connecting the two guides. We study both modes above and below the first cut-off for energy propagation down the coupled guide. The main tool used in the analysis is the so-called residue calculus technique in which complex variable theory is used to solve a system of equations which is derived from a mode-matching approach. For bound states below the first cut-off a single existence condition is derived, but for modes above this cut-off (but below the second cut-off), two conditions must be satisfied simultaneously. A number of results have been presented which show how the bound-state energies vary with the other parameters in the problem.
\end{abstract}

\footnotetext{
${ }^{1}$ Department of Mathematical Sciences, Loughborough University, LE11 3TU, UK (c.m.linton@lboro.ac.uk).
} 


\section{Introduction}

Over the past decade or so there have been a number of theoretical investigations into the phenomena of bound states in quantum waveguides, and acoustic resonances (or trapped modes) in acoustic waveguides. Though the two physical phenomena occur on vastly different scales, they are intimately related. It is our intention in this and the following article to show how some of the techniques that have been developed for the calculation of resonant frequencies in the acoustic context can be used to provide accurate and efficient tools for the evaluation of bound-sate energies in a particular class of quantum configurations.

Theoretical investigations into quantum wires and quantum waveguides became important when it became possible to manufacture crystalline structures of high purity within a semiconductor material which are of the order of tens of nanometres in size and on whose boundaries the wavefunctions are usually suppressed. As described in Duclos and Exner (1995), these properties make it reasonable to model the motion of an electron within such a microstructure as a free (spinless) particle in an infinite guide with a vanishing potential on the guide boundary. The underlying equation is of course the Schrödinger equation, but for stationary problems this reduces to the Helmholtz equation and thus the situation has direct analogues in the theories of acoustic and electromagnetic waves. Of interest here is the possible existence of non-trivial solutions which have finite energy. In quantum waveguides such solutions are known as bound states and their existence was explicitly demonstrated for the first time in Exner and Šeba (1989) for the case of a curved, thin planar strip and a review of early theoretical work on bound states in curved quantum waveguides (in both two and three dimensions) can be found in Duclos and Exner (1995). Some early experimental work, in which computed bound state energies were verified, is reported in Carini et al. (1992). The existence of bound states can have a significant influence on electron transport in mesoscopic systems.

In the acoustics literature, the same bounded solutions are typically referred to as acoustic resonances and the study of this phenomenon dates back to the experimental and theoretical work of Parker (1966, 1967). In Parker's setup the guide was a rigid duct, so the appropriate boundary conditions on the guide walls were of Neumann rather than Dirichlet type. Also, rather than being curved, the duct was perturbed by the inclusion of one or more splitter plates of finite length. Parker showed that such a geometry could support a non-trivial solution which was localized near to the plate(s) and which could be excited by vortex shedding from the plate edges when a mean flow of a certain speed was created in the duct. These resonances are extremely important in a number of engineering applications such as flow-induced vibration in cooler tubes and the design of turbomachinery; see Parker and Stoneman (1989) and the references cited therein.

Parker's modes were rediscovered in the early 1990s in a completely different physical situation: linear water waves in a wall-sided channel containing an obstacle. Here there are circumstances in which the depth dependence can be removed from the problem leaving 
the Helmholtz equation to be solved in a two-dimensional parallel-plate waveguide in which is situated a bounded obstacle. If this is a strip, then the problem is identical to the one considered by Parker. After some initial work showing that shapes other than plates could support bounded solutions (in this context usually called trapped modes) Evans, Levitin, and Vassiliev (1994) proved that this was the case for a very wide class of geometries.

The early work on these trapped modes focused on geometries which were symmetric about the guide centreline. This allows for a decomposition of the problem into independent symmetric and antisymmetric parts, the latter exhibiting a cut-off frequency below which it is impossible for energy to escape down the guide. In more formal language, the operator in the antisymmetric problem has a continuous spectrum which is bounded away form zero and the region below the continuous spectrum can be searched for discrete eigenvalues, corresponding to trapped modes. Perhaps the first function theoretic treatment of a problem of this type is that of Jones (1953) in which the author used comparison principles to prove the existence of discrete eigenvalues below the continuous spectrum for a class of semi-infinite guides.

The use of operator decomposition to create a gap below the continuous spectrum has proved extremely useful in the search for trapped modes/acoustic resonances and has been extended to cover a number of different situations; see, for example, Linton and McIver (1998), Groves (1998), Davies and Parnovski (1998). It is, however, merely a device which makes resonances relatively easy to find in certain situations, but it tells us nothing about the existence or otherwise of discrete eigenvalues embedded within the continuous spectra of the relevant operators. Little has been achieved for this more difficult problem, though some early progress is reported in Evans and Porter (1998), McIver et al. (2001) and McIver, Linton, and Zhang (2002) and this will be discussed in section 4 below.

The specific focus of our attention in this and the subsequent article is the study of coupled quantum waveguides and techniques that can be used to establish the existence of and compute the energies for bound states both in the discrete spectrum and embedded within the continuous spectrum. In two dimensions the guides we will consider consist of two straight parallel waveguides connected through a window in the common boundary. Experimental observations of a waveguide with a similar configuration can be found in Hirayama et al. (1992) and Hirayama et al. (1993). A numerical model, based on classical rebound effects in a straight wire for the experimental specification described in Hirayama et al. (1992), was provided in Takagaki and Ploog (1994).

One of the first theoretical treatments of resonances in coupled guides was that of Kunze (1993), who calculated the transmissivity of a wire coupled either to infinite space or to another wire via a small hole. Kunze showed that there are energies at which near resonances or quasibound states in the wire become possible and that these states cause a sharp downward dip in the conductance of the wire. However, it is the treatment given by Exner et al. (1996) which forms the starting point for our work. Exner et al. studied the bound states and scattering problems in parallel quantum waveguides of unequal width 
coupled laterally through a boundary window. The same type of variational argument as that employed by Evans, Levitin, and Vassiliev (1994) was used to show that the system always has at least one bound state for any window width. Moreover, the boundary-value problem was solved using a mode-matching technique, which allows one to construct the corresponding wave functions and determine how the bound-state energies depend on the parameters of the problem. Work by Bulla et al. (1997) showed that if the window was small enough so that only one simple eigenvalue below the continuous spectrum was present, this eigenvalue could be bounded from below and this result was extended by Exner and Vugalter (1996) who showed that for a sufficiently small window a two-sided asymptotic estimate for the gap between the continuous spectrum and this eigenvalue could be obtained. The case of a coupled waveguide containing a finite number of windows in the common boundary was considered in Exner and Vugalter (1997), who showed that if the windows are small enough there is just one isolated eigenvalue. Upper and lower bounds of the gap between this eigenvalue and the continuous spectrum were found using a variational approach. Other extensions to this type of analysis, including the incorporation of a magnetic field, or three laterally coupled guides, have been treated in Popov (2002) and Popov and Frolov (2003).

We treat the same geometry as that considered in Exner et al. (1996) with a coupled guide of total width $d$ formed by two parallel guides of width $b$ and $d-b$ linked via a window of length $2 a$ in their common boundary. After setting up the problem in section 2 we investigate the existence of bound states below the first cut-off for the coupled guide in section 3. Our starting point is a mode-matching analysis, but rather than solve the resulting system of equations numerically as in Exner et al. (1996), we use the so-called residue calculus technique described in Mittra and Lee (1971) to derive an approximate solution which is extremely accurate unless the $a / d$ is very small. This approximation also provides a very useful insight into how to solve the full problem in a numerically efficient way, and we show how an exponentially convergent linear system can be derived. Numerical results are presented and comparisons between the approximate and full solutions made. We find that bound states occur for any values of the parameters $a / d$ and $b / d$. We also use a variational argument to prove the existence of bound states for a sufficiently wide window and to provide estimates to the bound-state energies. It is shown that the upper bound of these estimates provides a very good approximation to the actual computed energies.

The residue calculus technique is readily adapted to the more difficult problem of searching for resonances above the first cut-off in the coupled guide, and bound states above the first cut-off and below the second cut-off are investigated in section 4 . The bound-state energies are shown to correspond to the intersection of two curves in parameter space and we find that the number of parameters which can be fixed is reduced by one. Thus if we fix the $b / d$, then bound states occur only for specific window widths. The possible existence of resonances between the second and third cut-off's is also discussed. Here we require the intersection of three lines and no solutions appear to exist. 


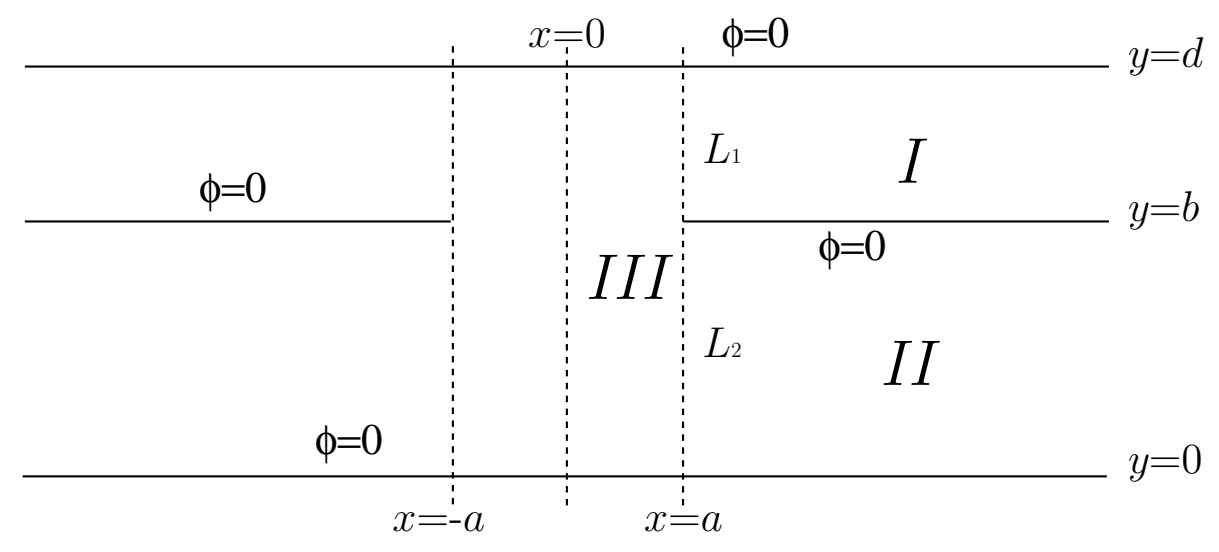

Figure 1: Definition sketch.

When the two guides which are coupled have the identical widths $(b / d=1 / 2)$ the resulting problem has an extra symmetry and this leads to some different results. Below the first cut-off there is essentially no difference and the results are just those obtained from the previous analysis in the limit as $b / d \rightarrow 1 / 2$, though the extra symmetry means that the equal-width problem could be solved rather more easily. However, above the cut-off we find that bound states are found in a different energy band, with those that were found for the unequal-width case disappearing as $b / d \rightarrow 1 / 2$. Some results for these new modes are presented.

\section{Formulation}

We consider a pair of two-dimensional waveguides of widths $b$ and $c$, coupled laterally through a window of width $2 a$ in their common boundary. The total width is $d=b+c$ and we assume without loss of generality that $b>d / 2$. The case when $b=d / 2$ can be treated more simply due to the extra symmetry in that case, and this in fact leads to a qualitative change in some of the results which will be explored in section 4. Cartesian axes are chosen so that the $x$-axis coincides with the lower boundary of the waveguide and the $y$-axis is chosen so that the waveguide is symmetric about the line $x=0$, as shown in Figure 1. Our geometry is thus defined by two non-dimensional parameters: the width of the window in the common boundary compared to the width of the whole guide, and the height of the window compared to the width of the full coupled guide $(2 a / d$ and $b / d$, respectively).

Initially we shall seek a solution which is even (symmetric) about $x=0$, by considering the region $x>0$ and seeking a function $\phi(x, y)$ which satisfies

$$
\frac{\partial \phi}{\partial x}=0 \text { on } x=0,0<y<d .
$$


The function $\phi(x, y)$ must also satisfy the Helmholtz equation within the waveguide

$$
\left(\nabla^{2}+k^{2}\right) \phi=0, \quad 0<y<d \text { except on } y=b, x>a .
$$

In the acoustic context $k=\omega / c$ where $\omega$ is the frequency of oscillation and $c$ is the speed of sound, whereas in the related quantum mechanical problem $k^{2}=2 m E / \hbar^{2}$, in which $m$ is the mass of the electron, and $E$ is its total energy. In what follows, the parameter $k$ will be referred to as the energy. The function $\phi$ satisfies Dirichlet boundary conditions on the waveguide walls,

$$
\begin{aligned}
& \phi=0 \text { on } y=0, x>0, \\
& \phi=0 \text { on } y=b, x>a, \\
& \phi=0 \text { on } y=d, x>0,
\end{aligned}
$$

and a radiation condition specifying that no waves propagate out to infinity,

$$
\phi \rightarrow 0 \text { as } x \rightarrow \infty,
$$

which can be shown (using Green's theorem) to be equivalent in this problem to the statement that $\phi$ must possess finite energy:

$$
\int_{\Omega}|\nabla \phi|^{2} \mathrm{~d} \Omega<\infty
$$

where $\Omega$ is the interior of the coupled guide. We finally assume $\phi$ is non-singular and that

$$
\nabla \phi=O\left(r^{-\frac{1}{2}}\right) \text { as } r \equiv\left\{(x-a)^{2}+(y-b)^{2}\right\}^{\frac{1}{2}} \rightarrow 0,
$$

anticipating a singularity in the derivative of $\phi$ at the edge of the window.

It is useful to split the domain into three regions as shown in Figure 1. Region $I$ is $b<y<d, x>a$, region $I I$ is $0<y<b, x>a$, and region $I I I$ is $0<y<d, 0<x<a$, We can represent the function $\phi$ by a function $\phi_{i}(i=1,2,3)$ in each region, with the following continuity conditions applied at the boundaries between the regions:

$$
\phi_{i}=\phi_{3}, \quad \frac{\partial \phi_{i}}{\partial x}=\frac{\partial \phi_{3}}{\partial x}, \quad \text { on } L_{i}, i=1,2,
$$

where $L_{1}$ is $x=a, b<y<d, L_{2}$ is $x=a, 0<y<b$ and we will write $L_{3}$ for $L_{1} \cup L_{2}$. We then introduce the complete orthogonal sets

$$
\begin{array}{lll}
\Psi_{n}^{(1)}(y)=2^{1 / 2} \sin \nu_{n}(d-y), & \nu_{n}=n \pi / c, & n \in \mathbb{N}, \\
\Psi_{n}^{(2)}(y)=2^{1 / 2} \sin \mu_{n}(b-y), & \mu_{n}=n \pi / b, & n \in \mathbb{N}, \\
\Psi_{n}^{(3)}(y)=2^{1 / 2} \sin \lambda_{n}(d-y), & \lambda_{n}=(n+1) \pi / d, & n \in \mathbb{N}_{0},
\end{array}
$$

which satisfy

$$
\frac{1}{\left|L_{i}\right|} \int_{L_{i}} \Psi_{n}^{(i)}(y) \Psi_{m}^{(i)}(y) \mathrm{d} y=\delta_{m n}, \quad i=1,2,3
$$


where $\delta_{m n}$ is the Kronecker delta. Here and throughout we use the symbol $\mathbb{N}$ for the set $\{1,2,3, \ldots\}$ and the symbol $\mathbb{N}_{0}$ for the set $\{0,1,2, \ldots\}$

Separation of variables shows that the eigenfunction expansions for the three regions can be written

$$
\begin{array}{lll}
\phi_{1}(x, y) & =\sum_{n=1}^{\infty} U_{n}^{(1)} \frac{\mathrm{e}^{-\alpha_{n}(x-a)}}{-\alpha_{n}} \Psi_{n}^{(1)}(y), & \alpha_{n}=\left(\nu_{n}^{2}-k^{2}\right)^{1 / 2}, \\
\phi_{2}(x, y)=\sum_{n=1}^{\infty} U_{n}^{(2)} \frac{\mathrm{e}^{-\beta_{n}(x-a)}}{-\beta_{n}} \Psi_{n}^{(2)}(y), & \beta_{n}=\left(\mu_{n}^{2}-k^{2}\right)^{1 / 2}, \\
\phi_{3}(x, y)=\sum_{n=0}^{\infty} U_{n}^{(3)} \frac{\cosh \gamma_{n} x}{\gamma_{n} \sinh \gamma_{n} a} \Psi_{n}^{(3)}(y), & \gamma_{n}=\left(\lambda_{n}^{2}-k^{2}\right)^{1 / 2},
\end{array}
$$

where $U_{n}^{(i)}, i=1,2,3$, are unknown complex constants and various factors have been introduced for convenience. If we restrict the energy by

$$
k b<\pi
$$

then, since $b>d / 2$, the values of $\alpha_{n}$ and $\beta_{n}, n \in \mathbb{N}$ will all be real and positive. As these terms appear in the eigenfunction expansions as coefficients of negative exponentials, the restriction on energy produces exponential decay down the guide in both regions $I$ and $I I$. We define $k b=\pi$ as the first cut-off of the coupled waveguide and consider the case of bound states whose energies are below this cut-off.

\section{Bound states below the first cut-off}

If $k b<\pi$ then $\alpha_{n}, \beta_{n}$, and $\gamma_{n}, n \in \mathbb{N}$, are all real and positive. However, provided $k d>\pi$, $\gamma_{0}$ will be purely imaginary and the corresponding mode will be oscillatory in region III. We thus anticipate that a necessary condition for the existence of trapped modes will be

$$
\pi<k d<\frac{d \pi}{b} .
$$

If we apply the continuity conditions (2.9) we obtain

$$
\sum_{n=0}^{\infty} U_{n}^{(3)} \frac{\operatorname{coth} \gamma_{n} a}{\gamma_{n}} \Psi_{n}^{(3)}(y)= \begin{cases}\sum_{n=1}^{\infty} U_{n}^{(1)} \frac{\Psi_{n}^{(1)}(y)}{-\alpha_{n}}, & y \in L_{1}, \\ \sum_{n=1}^{\infty} U_{n}^{(2)} \frac{\Psi_{n}^{(2)}(y)}{-\beta_{n}}, & y \in L_{2},\end{cases}
$$

and

$$
\sum_{n=0}^{\infty} U_{n}^{(3)} \Psi_{n}^{(3)}(y)= \begin{cases}\sum_{n=1}^{\infty} U_{n}^{(1)} \Psi_{n}^{(1)}(y), & y \in L_{1}, \\ \sum_{n=1}^{\infty} U_{n}^{(2)} \Psi_{n}^{(2)}(y), & y \in L_{2} .\end{cases}
$$


We can convert (3.2) and (3.3) into an infinite system of linear algebraic equations by multiplying each by $\Psi_{m}^{(3)}, m \in \mathbb{N}_{0}$, and integrating over $L_{3}$. This leads to

$$
\begin{array}{rlrl}
U_{m}^{(3)} & =\sum_{n=1}^{\infty} U_{n}^{(1)} d_{n m}+\sum_{n=1}^{\infty} U_{n}^{(2)} e_{n m}, \quad m \in \mathbb{N}_{0}, \\
U_{m}^{(3)} \frac{\operatorname{coth} \gamma_{m} a}{\gamma_{m}} & =\sum_{n=1}^{\infty} \frac{U_{n}^{(1)}}{-\alpha_{n}} d_{n m}+\sum_{n=1}^{\infty} \frac{U_{n}^{(2)}}{-\beta_{n}} e_{n m}, & m \in \mathbb{N}_{0},
\end{array}
$$

where we have defined

$$
\begin{array}{lll}
d_{n m}=\frac{1}{d} \int_{L_{1}} \Psi_{n}^{(1)}(y) \Psi_{m}^{(3)}(y) \mathrm{d} y=\frac{2 \nu_{n}(-1)^{n} \sin \lambda_{m} c}{d\left(\gamma_{m}^{2}-\alpha_{n}^{2}\right)}, & & m \in \mathbb{N}_{0}, n \in \mathbb{N}, \\
e_{n m}=\frac{1}{d} \int_{L_{2}} \Psi_{n}^{(2)}(y) \Psi_{m}^{(3)}(y) \mathrm{d} y=\frac{2 \mu_{n} \sin \lambda_{m} c}{d\left(\gamma_{m}^{2}-\beta_{n}^{2}\right)}, & & m \in \mathbb{N}_{0}, n \in \mathbb{N} .
\end{array}
$$

It is necessary that $\gamma_{m} \neq \alpha_{n}$ and $\gamma_{m} \neq \beta_{n}$ for any $n$ and $m$ and we can ensure this by assuming that $b / d$ is irrational, although Evans, Linton, and Ursell (1993), Appendix D, show how continuity arguments can be used to remove this restriction so that the conditions for bound states that are obtained are valid for all values of $b / d$.

Eliminating $U_{m}^{(3)}$ from (3.4) and (3.5), and substituting for $d_{n m}$ and $e_{n m}$ from (3.6) and (3.7), we can derive

$$
\sum_{n=1}^{\infty} U_{n}\left(\frac{1}{\alpha_{n}-\gamma_{m}}+\frac{\zeta_{m}}{\alpha_{n}+\gamma_{m}}\right)-\sum_{n=1}^{\infty} V_{n}\left(\frac{1}{\beta_{n}-\gamma_{m}}+\frac{\zeta_{m}}{\beta_{n}+\gamma_{m}}\right)=0, \quad m \in \mathbb{N}_{0},
$$

where we have defined

$$
U_{n}=\frac{U_{n}^{(1)}(-1)^{n} \nu_{n}}{\alpha_{n}}, \quad V_{n}=\frac{U_{n}^{(2)} \mu_{n}}{\beta_{n}}, \quad \zeta_{m}=\mathrm{e}^{-2 \gamma_{m} a} .
$$

So far we have only used the boundary conditions (2.1)-(2.6) and not the condition (2.8), which anticipates the singular behaviour near the edge. Since $\sum_{n=1}^{\infty} n^{\tau} e^{-n x}=$ $O\left(x^{-1-\tau}\right)$ as $x \rightarrow 0+$ (see, e.g. Martin 1995), consideration of the gradient of $\phi$ near the edge shows that

$$
U_{n}, V_{n}=O\left(n^{-\frac{1}{2}}\right) \text { as } n \rightarrow \infty
$$

\section{Approximate solution}

We now derive an approximate solution to (3.8) for large $a$, taking into account the asymptotic behaviour required from (3.10). Note that since $\gamma_{m}, m \in \mathbb{N}$, is real, the terms $\zeta_{m}, m \in \mathbb{N}$, appearing in (3.8) decay rapidly to zero as $a / d \rightarrow \infty$. A good approximation for large $a$ is therefore to set $\zeta_{m}=0$ for $m \in \mathbb{N}$. The idea for this type of approximation (which is equivalent to assuming that the two edges at $x= \pm a, y=b$ can be 
treated independently) goes back to Hurd (1954), who was studying the propagation of electromagnetic surface waves along a comb grating. We would then have

$$
\sum_{n=1}^{\infty}\left(\frac{U_{n}}{\alpha_{n}-\gamma_{m}}-\frac{V_{n}}{\beta_{n}-\gamma_{m}}\right)=-\delta_{m 0} \zeta_{0} \sum_{n=1}^{\infty}\left(\frac{U_{n}}{\alpha_{n}+\gamma_{0}}-\frac{V_{n}}{\beta_{n}+\gamma_{0}}\right), \quad m \in \mathbb{N}_{0},
$$

a system of equations that can be solved explicitly using a method originally described in Whitehead (1951) and Berz (1951).

Consider the quantities

$$
I_{m}=\lim _{N \rightarrow \infty} \frac{1}{2 \pi \mathrm{i}} \int_{C_{N}} \frac{f(z)}{z-\gamma_{m}} \mathrm{~d} z, \quad J_{m}=\lim _{N \rightarrow \infty} \frac{1}{2 \pi \mathrm{i}} \int_{C_{N}} \frac{f(z)}{z+\gamma_{m}} \mathrm{~d} z, \quad m \in \mathbb{N}_{0},
$$

where $C_{N}$ is a sequence of contours (to be determined) on which $z \rightarrow \infty$ as $N \rightarrow \infty$ and $f(z)$ is a meromorphic function which is assumed to have the following properties:

P1. $f(z)$ has simple poles at $z=\alpha_{n}$ and $z=\beta_{n}, n \in \mathbb{N}$,

P2. $f(z)$ has simple zeros at $z=\gamma_{n}, n \in \mathbb{N}$, but not at $z=\gamma_{0}$,

P3. $f(z)=o(1)$ as $|z| \rightarrow \infty$ on $C_{N}$ as $N \rightarrow \infty$.

Condition P3 ensures that $I_{m}=0$ and $J_{m}=0$. Applying Cauchy's residue theorem to the integrals in (3.12) we find

$$
\begin{gathered}
\sum_{n=1}^{\infty} \frac{R\left(f: \alpha_{n}\right)}{\alpha_{n}-\gamma_{m}}+\sum_{n=1}^{\infty} \frac{R\left(f: \beta_{n}\right)}{\beta_{n}-\gamma_{m}}+\delta_{m 0} f\left(\gamma_{0}\right)=0 \\
\sum_{n=1}^{\infty} \frac{R\left(f: \alpha_{n}\right)}{\alpha_{n}+\gamma_{m}}+\sum_{n=1}^{\infty} \frac{R\left(f: \beta_{n}\right)}{\beta_{n}+\gamma_{m}}+f\left(-\gamma_{m}\right)=0
\end{gathered}
$$

where $R\left(f: z_{0}\right)$ represents the residue of $f(z)$ at $z=z_{0}$, and $m \in \mathbb{N}_{0}$, in each case. If we normalise $f(z)$ by setting

$$
f\left(\gamma_{0}\right)=1,
$$

then a comparison of (3.13) and (3.14) with (3.11) shows that the required solution of (3.11) is given by

$$
U_{n}=R\left(f: \alpha_{n}\right), \quad V_{n}=-R\left(f: \beta_{n}\right), \quad n \in \mathbb{N},
$$

provided (3.10) is satisfied and

$$
f\left(-\gamma_{0}\right) \zeta_{0}=-1
$$

If we can determine a suitable $f(z)$ and sequence of contours $C_{N}$ then (3.17) is the condition for the existence of bound states.

The method by which $f(z)$ can be constructed follows closely that given in Evans, Linton, and Ursell (1993) and we will simply note here that the a sequence of contours $C_{N}$ can be constructed so that the function

$$
f(z)=h_{1} g(z),
$$


where

$$
\begin{aligned}
h_{1} & =\exp \left\{\frac{\gamma_{0}}{\pi}(b \ln (d / b)+c \ln (d / c))\right\} \prod_{n=1}^{\infty} \frac{\left(1-\gamma_{0} / \alpha_{n}\right)\left(1-\gamma_{0} / \beta_{n}\right)}{\left(1-\gamma_{0} / \gamma_{n}\right)} \\
g(z) & =\exp \left\{\frac{-z}{\pi}(b \ln (d / b)+c \ln (d / c))\right\} \prod_{n=1}^{\infty} \frac{\left(1-z / \gamma_{n}\right)}{\left(1-z / \alpha_{n}\right)\left(1-z / \beta_{n}\right)},
\end{aligned}
$$

has all the necessary properties. In fact $f(z)=O\left(z^{-\frac{1}{2}}\right)$ as $|z| \rightarrow \infty$ on $C_{N}$ as $N \rightarrow \infty$. Note that the fact that $\gamma_{n} \sim(n+1) \pi / d$ rather than $n \pi / d$ (while $\alpha_{n} \sim n \pi / c$ and $\beta_{n} \sim$ $n \pi / b)$ is crucial.

The condition for the existence of bound states (3.17) is

$$
f\left(\mathrm{i} \gamma^{\prime}\right)=-\mathrm{e}^{-2 \mathrm{i} \gamma^{\prime} a},
$$

where

$$
\gamma^{\prime}=\mathrm{i} \gamma_{0}=\left(k^{2}-(\pi / d)^{2}\right)^{\frac{1}{2}}
$$

and this reduces to

$$
\gamma^{\prime}(a-\Theta)=\chi+\left(n-\frac{1}{2}\right) \pi, \quad n \text { an integer },
$$

where

$$
\begin{aligned}
& \Theta=\frac{1}{\pi}(b \ln (d / b)+c \ln (d / c)), \\
& \chi=\sum_{n=1}^{\infty}\left(\tan ^{-1}\left(\frac{\gamma^{\prime}}{\gamma_{n}}\right)-\tan ^{-1}\left(\frac{\gamma^{\prime}}{\alpha_{n}}\right)-\tan ^{-1}\left(\frac{\gamma^{\prime}}{\beta_{n}}\right)\right) .
\end{aligned}
$$

For the case of antisymmetry about $x=0$, condition (2.1) is replaced by

$$
\phi=0 \text { on } x=0,0<y<d,
$$

and the system of equations (3.8) becomes

$$
\sum_{n=1}^{\infty} U_{n}\left(\frac{1}{\alpha_{n}-\gamma_{m}}-\frac{\zeta_{m}}{\alpha_{n}+\gamma_{m}}\right)-\sum_{n=1}^{\infty} V_{n}\left(\frac{1}{\beta_{n}-\gamma_{m}}-\frac{\zeta_{m}}{\beta_{n}+\gamma_{m}}\right)=0, \quad m \in \mathbb{N}_{0} .
$$

The approximate existence condition (3.21) becomes

$$
f\left(\mathrm{i} \gamma^{\prime}\right)=\mathrm{e}^{-2 \mathrm{i} \gamma^{\prime} a}
$$

with exactly the same function $f$ as before, and this reduces to

$$
\gamma^{\prime}(a-\Theta)=\chi+n \pi, \quad n \text { an integer, }
$$

where $\Theta$ and $\chi$ are defined as before. 


\section{Full solution}

The approximate solution derived above can be used as the basis for a very efficient numerical solution to the problem. The ideas behind the method are described in detail in Mittra and Lee (1971), and briefly in Jones (1994), §2.12. We form a solution to (3.8) by considering the quantities

$$
I_{m}=\lim _{N \rightarrow \infty} \frac{1}{2 \pi \mathrm{i}} \int_{C_{N}} f(z)\left(\frac{1}{z-\gamma_{m}}+\frac{\zeta_{m}}{z+\gamma_{m}}\right) \mathrm{d} z, \quad m \in \mathbb{N}_{0},
$$

where now $f(z)$ must satisfy

P1. $f(z)$ has simple poles at $z=\alpha_{n}$ and $z=\beta_{n}, n \in \mathbb{N}$,

P2. $f(z)=o(1)$ as $|z| \rightarrow \infty$ on $C_{N}$ as $N \rightarrow \infty$.

We now let

$$
f(z)=g(z) h(z)
$$

where $g(z)$ is given by $(3.20)$ and

$$
h(z)=1+\sum_{n=1}^{\infty} \frac{A_{n}}{z-\gamma_{n}}
$$

for some unknown constants $A_{n}$. The function $h(z)$ is chosen to cancel the zeros of $g(z)$ at $\gamma_{n}$, and we anticipate that as $a \rightarrow \infty, h(z)$ will tend rapidly to 1 , since the exponential terms $\zeta_{n}$ will decay rapidly to zero. Thus the constants $A_{m}$ will also tend to zero rapidly as $a \rightarrow \infty$.

If we apply Cauchy's residue theorem to the integral in (3.30) we obtain

$$
\begin{aligned}
\sum_{n=1}^{\infty} R\left(f: \alpha_{n}\right)\left(\frac{1}{\alpha_{n}-\gamma_{m}}+\frac{\zeta_{m}}{\alpha_{n}+\gamma_{m}}\right)+ & \sum_{n=1}^{\infty} R\left(f: \beta_{n}\right)\left(\frac{1}{\beta_{n}-\gamma_{m}}+\frac{\zeta_{m}}{\beta_{n}+\gamma_{m}}\right) \\
& +f\left(\gamma_{m}\right)+\zeta_{m} f\left(-\gamma_{m}\right)=0, \quad m \in \mathbb{N}_{0}
\end{aligned}
$$

Comparing (3.33) with (3.8) we see that $U_{n}=R\left(f: \alpha_{n}\right), V_{n}=-R\left(f: \beta_{n}\right), n \in \mathbb{N}$, provided (3.10) is satisfied and

$$
f\left(\gamma_{m}\right)+\zeta_{m} f\left(-\gamma_{m}\right)=0, \quad m \in \mathbb{N}_{0}
$$

For $m \in \mathbb{N}$ this is equivalent to an infinite system of equations for the unknowns $A_{m}$ :

$$
A_{m}+B_{m} \sum_{n=1}^{\infty} \frac{A_{n}}{\gamma_{m}+\gamma_{n}}=B_{m}, \quad m \in \mathbb{N}
$$

where

$$
B_{m}=2 \gamma_{m} \mathrm{e}^{2 \gamma_{m}(\Theta-a)} \frac{\left(\alpha_{m}-\gamma_{m}\right)\left(\beta_{m}-\gamma_{m}\right)}{\left(\alpha_{m}+\gamma_{m}\right)\left(\beta_{m}+\gamma_{m}\right)} \prod_{\substack{n=1 \\ n \neq m}}^{\infty} \frac{\left(1+\gamma_{m} / \gamma_{n}\right)\left(1-\gamma_{m} / \alpha_{n}\right)\left(1-\gamma_{m} / \beta_{n}\right)}{\left(1+\gamma_{m} / \alpha_{n}\right)\left(1+\gamma_{m} / \beta_{n}\right)\left(1-\gamma_{m} / \gamma_{n}\right)}
$$


Unlike (3.8) this system of equations is real and the presence of the factor $\zeta_{m}$ in $B_{m}$ shows that as $a \rightarrow \infty$, the coefficients $A_{m}$ decay exponentially to zero. Moreover, the terms $B_{m}$ decay exponentially as $m \rightarrow \infty$ and so the system can be solved very efficiently via a numerical truncation technique. It can be proved, using the methods described in Evans (1992), Appendix B, and Evans, Linton, and Ursell (1993), Appendix C, that the infinite system (3.35) has a unique solution $A_{n}$ with $\sum_{n=1}^{\infty} A_{n}^{2}<\infty$ provided $a / d$ is sufficiently large.

We now return to the one condition still to be satisfied, namely (3.34) with $m=0$. This is

$$
\mathrm{e}^{2 \mathrm{i} \gamma^{\prime} a}=-\frac{f\left(-\mathrm{i} \gamma^{\prime}\right)}{f\left(\mathrm{i} \gamma^{\prime}\right)}
$$

Now, since $A_{n}$ and $\gamma_{n}$ are real for $n \in \mathbb{N}$ we can write

$$
\frac{h\left(-\mathrm{i} \gamma^{\prime}\right)}{h\left(\mathrm{i} \gamma^{\prime}\right)}=\mathrm{e}^{2 \mathrm{i} \sigma}
$$

where

$$
\sigma=\arg \left(h\left(-\mathrm{i} \gamma^{\prime}\right)\right)=\arg \left(1-\sum_{n=1}^{\infty} \frac{A_{n}}{\gamma_{n}+\mathrm{i} \gamma^{\prime}}\right) .
$$

Then, with $\chi$ and $\Theta$ as before, the condition for the existence of trapped modes (3.37) reduces to

$$
\gamma^{\prime}(a-\Theta)=\chi+\sigma+\left(n-\frac{1}{2}\right) \pi, \quad n \text { an integer. }
$$

This condition differs from the approximate condition (3.23) by the inclusion of the term $\sigma$. This depends on $a / d$, but it is clear that as $a / d \rightarrow \infty, \sigma \rightarrow \arg (1)=0$.

For the case of antisymmetry about $x=0,(3.34)$ becomes

$$
f\left(\gamma_{m}\right)-\zeta_{m} f\left(-\gamma_{m}\right)=0, \quad m \in \mathbb{N}_{0}
$$

with the infinite system of equations equivalent to (3.35) being

$$
A_{m}-B_{m} \sum_{n=1}^{\infty} \frac{A_{n}}{\gamma_{m}+\gamma_{n}}=-B_{m} \quad m \in \mathbb{N},
$$

and $B_{m}$ is given by (3.36) as before. The condition for antisymmetric modes, equivalent to $(3.40)$, is thus

$$
\gamma^{\prime}(a-\Theta)=\chi+\sigma^{\prime}+n \pi, \quad n \text { an integer, }
$$

where $\sigma^{\prime}$ is the argument of $h\left(-\mathrm{i} \gamma^{\prime}\right)$ with the constants $A_{n}$ given by the solution of (3.42).

\section{Results}

The systems of equations (3.35) and (3.42) need to be solved by truncation but due to the exponential convergence of the $B_{m}$ terms only a small truncation parameter is needed. 


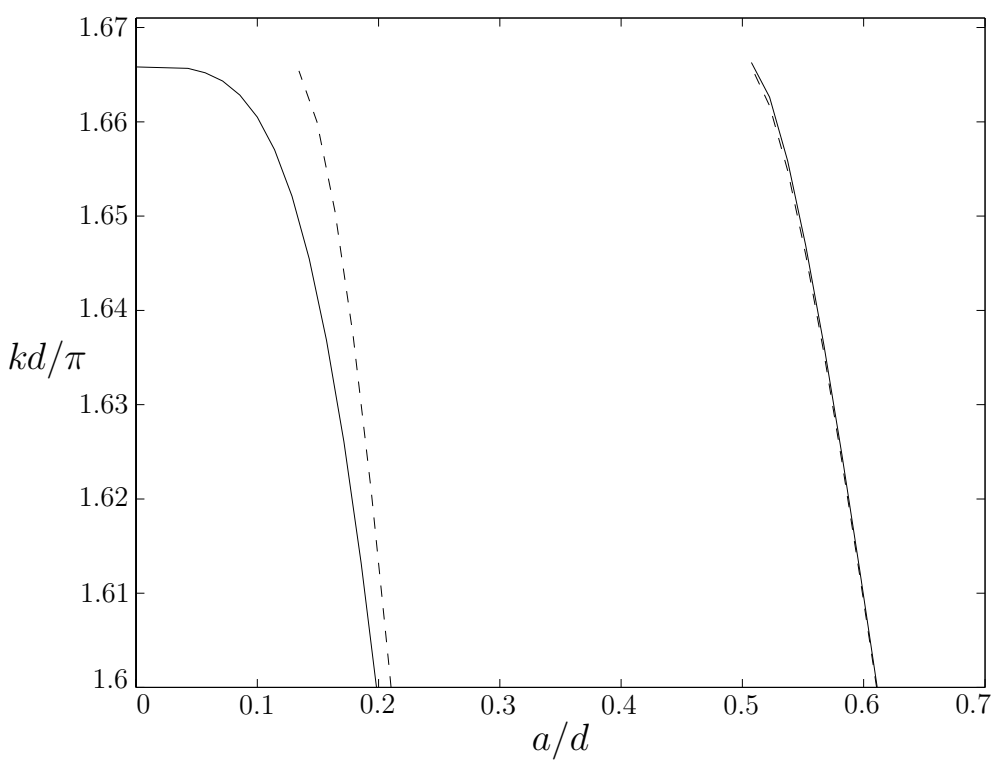

Figure 2: A comparison of the bound-state energies, $k d / \pi$, for modes symmetric and antisymmetric about $x=0$ for the approximate solution (dashed) and the full solution (solid) plotted against $a / d$ when $b / d=0.6$.

In the following results a truncation parameter of 5 was used to assure high accuracy for all parameter values, but a $2 \times 2$ system is adequate unless $a / d$ is very small. Figure 2 shows results for $b / d=0.6$ and compares the non-dimensional bound-state energies, $k d / \pi$, computed from the approximate solution (dashed lines) with those found from the full solution (solid lines). The two curves on the left of the figure correspond to the first symmetric (about $x=0$ ) mode and the other two correspond to the first antisymmetric mode. For all but the smallest values of $a / d$, the results computed from the full and approximate methods are indistinguishable. Only when $a / d$ is less than about 0.5 do the full solution and the approximate solution produce significantly different results.

In Figure 3 a typical set of trapped-mode wavenumbers, $k d / \pi$, computed from the full solutions (3.40) and (3.43), are plotted against $a / d$, for $b / d=0.75$. The solid lines correspond to modes symmetric about $x=0$ and the dashed lines correspond to modes antisymmetric about $x=0$. We see that as $a / d$ increases the number of modes present also increases, and the modes appear alternatively symmetric and antisymmetric from the cut-off $k d=d \pi / b=4 \pi / 3$, and decrease towards $k d=\pi$.

The variation of bound-state energies with $b / d$ when $a / d=3$ is shown in Figure 4 . The solid lines represent symmetric modes, the dashed lines correspond to antisymmetric modes, and the dotted line represents the upper cut-off $k b=\pi$. It is clear that as $b / d$ increases from 0.5 , the number of modes present decreases and the energy of each of the modes decreases slightly. The figure shows that modes are present for any value of $b / d$ in the interval $[0.5,1)$. 


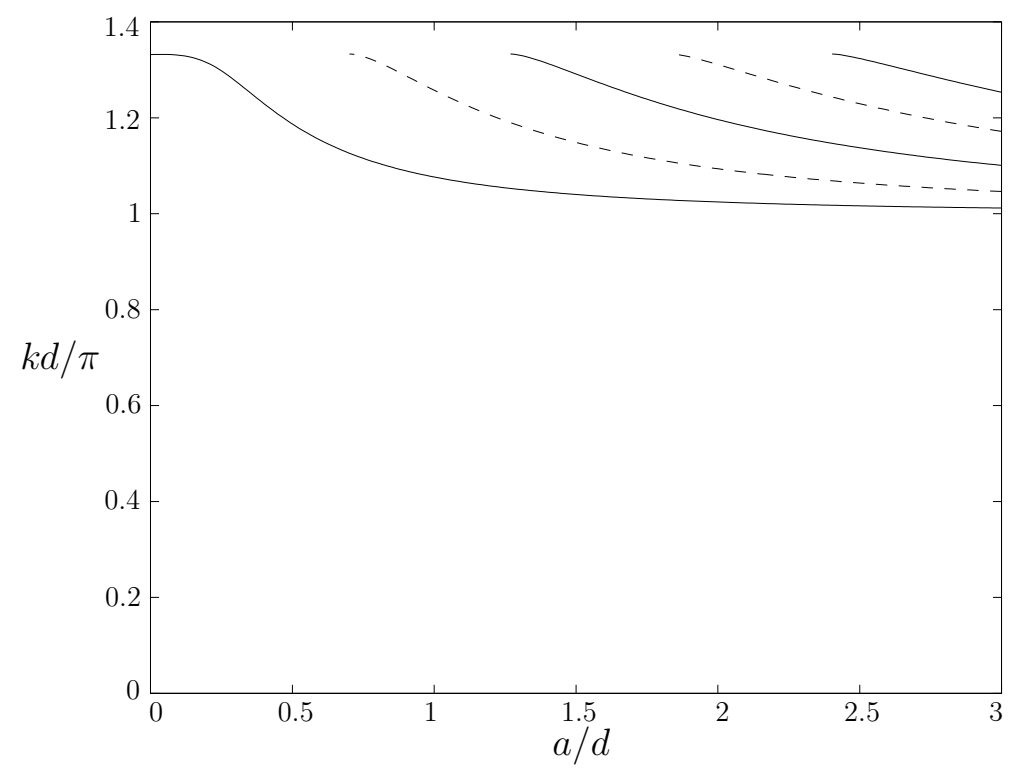

Figure 3: Bound-state energies, $k d / \pi$, for modes symmetric $(-)$ and antisymmetric $(--)$ about $x=0$, plotted against $a / d$ when $b / d=0.75$.

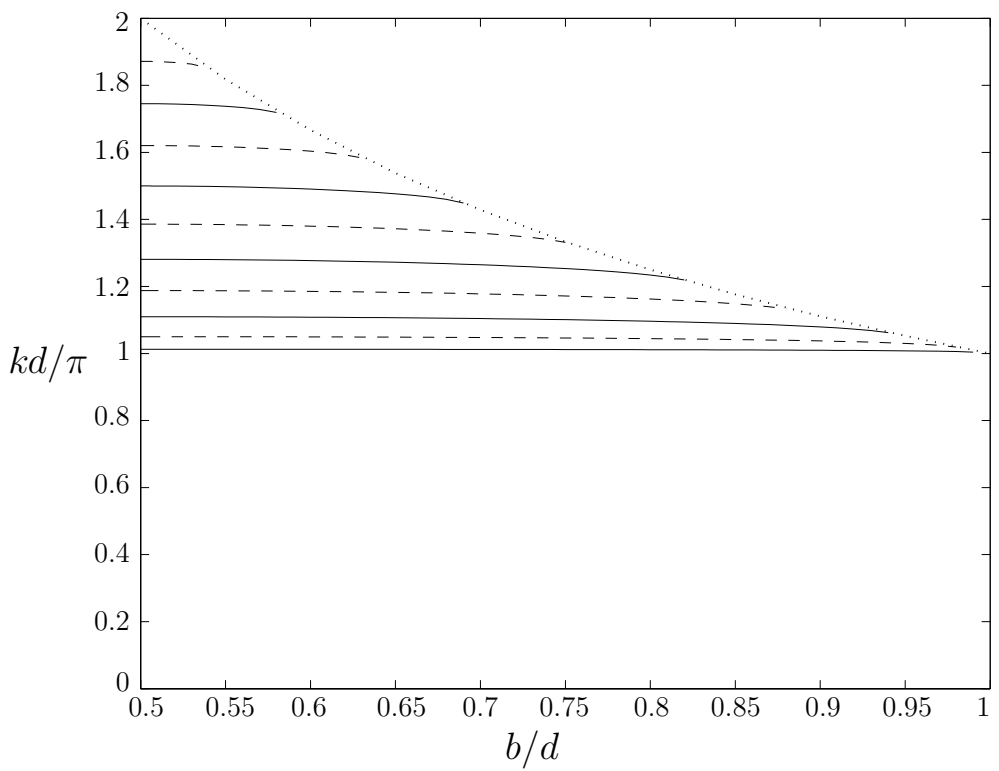

Figure 4: Bound-state energies, $k d / \pi$, for modes symmetric $(-)$ and antisymmetric $(--)$ about $x=0$, plotted against $b / d$ when $a / d=3$. The dotted line is the curve $k b=\pi$. 


\section{Variational methods}

Trapped modes occuring in two-dimensional acoustic waveguides containing long obstacles symmetric about the centreline were considered by Khallaf, Parnovski, and Vassiliev (2000). In their paper the authors use variational arguments to provide estimates for the trapped mode frequencies and also to prove that the number of trapped modes occuring is asymptotically proportional to the obstacle's length. We will extend their method to provide estimates for the bound-state energies in our problem and to prove existence for a sufficiently large window. The main difference between the two problems is that the laterally coupled guide under consideration here is not symmetric about the centreline of the waveguide.

Our waveguide can be thought of as an unbounded domain $\Omega$ defined as

$$
\Omega=\{(0, \infty) \times(0, d)\} \backslash\{[a, \infty) \times[b]\},
$$

where $a>0$ and $\frac{1}{2}<b / d<1$. We are trying to find estimates for values of $\lambda$ for which there is a non-trivial solution $\phi(x, y)$ to the boundary-value problem

$$
-\nabla^{2} \phi=\lambda \phi \text { in } \Omega
$$

subject to the boundary conditions (2.3)-(2.6), and (2.1) for symmetric modes or (3.26) for antisymmetric modes. In other words we seek the eigenvalues of $-\nabla^{2}$ (defined on an appropriate domain) and since wave-like modes can propagate to infinity if $k b>\pi$, the continuous spectrum for either the symmetric or the antisymmetric problem is the semi-interval $\left[\pi^{2} / b^{2}, \infty\right)$.

We consider the Rayleigh quotient defined by

$$
Q(\phi)=\frac{\int_{\Omega}|\nabla \phi|^{2} \mathrm{~d} \Omega}{\int_{\Omega}|\phi|^{2} \mathrm{~d} \Omega},
$$

and set

$$
\lambda_{1}=\inf _{\phi \in \mathcal{H}_{0}^{1} \backslash\{0\}} Q(\phi) .
$$

(The symbol $\lambda_{n}$ has a different meaning in this section than elsewhere in the paper.) Here $\mathcal{H}_{0}^{1}$ is the Sobolev space consisting of all functions in $L^{2}(\Omega)$ which also have squareintegrable first partial derivatives and which vanish on the boundary of $\Omega$ at the same places that the solution $\phi$ does. In particular, the symbol $\mathcal{H}_{0}^{1}$ represents different spaces in the symmetric and antisymmetric problems, since in the latter case functions in $\mathcal{H}_{0}^{1}$ must vanish on $x=0,0<y<d$. It is a standard result (see, e.g., Edmunds and Evans 1987, chapter XI) that $\lambda_{1}>0$ is the lowest point of the spectrum of our operator and if $\lambda_{1}<\pi^{2} / b^{2}$, then $\lambda_{1}$ must be an eigenvalue of the problem (the smallest) and the function $\varphi_{1}$ for which $Q\left(\varphi_{1}\right)=\lambda_{1}$ is the associated eigenfunction (bound state). We can define

$$
\lambda_{n}=\inf _{\substack{\phi \in \mathcal{H}_{1}^{1} \backslash\{0\} \\ \phi \perp \varphi_{i}, i=1,2, \ldots, n-1}} Q(\phi)
$$


and if $\lambda_{n}<\pi^{2} / b^{2}, \lambda_{n}$ is the $n$th eigenvalue for the problem (arranged in order of increasing size and accounting for multiplicity). If $\lambda_{n+1}=\pi^{2} / b^{2}$, there will be exactly $n$ eigenvalues below the first cut-off. We label the eigenvalues below the continuous spectrum as $\lambda_{j, s}$ for the symmetric problem (and we assume that there are $n_{s}$ of them) and $\lambda_{j, a}$ for the antisymmetric problem (of which there are $n_{a}$ ).

We now use the idea of Dirichlet-Neumann bracketing (see, e.g., Courant and Hilbert 1953) which is a consequence of the variational principle outlined above. The domain $\Omega$ is split into the regions $I, I I$ and $I I I$ defined previously and we consider the eigenvalues associated with each region separately when an artificial boundary condition is imposed on $L_{3}$. We denote the $j$ th eigenvalue occuring in region $i$ with a Dirichlet (resp. Neumann) condition on $L_{3}$ as $\lambda_{j}^{i, D}$, (resp. $\lambda_{j}^{i, N}$ ). We also define a counting function $N^{i, D}(\lambda)$, (resp. $N^{i, N}(\lambda)$ ), as the number of eigenvalues less than $\lambda$ in region $i$, with a Dirichlet (resp. Neumann) condition on $L_{3}$. An extra subscript ( $s$ or $a$ ) will be used whenever it is necessary to distinguish between the symmetric and antisymmetric problems. If $\lambda<\pi^{2} / b^{2}$ we have

$$
N^{I, D}(\lambda)+N^{I I, D}(\lambda)+N_{s}^{I I I, D}(\lambda) \leq N_{s}(\lambda) \leq N^{I, N}(\lambda)+N^{I I, N}(\lambda)+N_{s}^{I I I, N}(\lambda),
$$

where $N_{s}(\lambda)$ is the total number of eigenvalues below $\lambda$ for the symmetric problem. Note that $N_{s}\left(\pi^{2} / b^{2}\right)=n_{s}$.

A straightforward calculation shows that

$$
\begin{aligned}
& \left\{\lambda_{j, s}^{I I I, D}\right\}_{j=1}^{\infty}=\left\{(n-1 / 2)^{2} \pi^{2} / a^{2}+(m \pi)^{2} / d^{2}\right\}_{n, m=1}^{\infty} \\
& \left\{\lambda_{j, s}^{I I I, N}\right\}_{j=1}^{\infty}=\left\{(n-1)^{2} \pi^{2} / a^{2}+(m \pi)^{2} / d^{2}\right\}_{n, m=1}^{\infty},
\end{aligned}
$$

and that there are no eigenvalues in regions $I$ or $I I$ and so (3.49) becomes

$$
N_{s}^{I I I, D}(\lambda) \leq N_{s}(\lambda) \leq N_{s}^{I I I, N}(\lambda),
$$

provided $\lambda<\pi^{2} / b^{2}$. It then follows (see, e.g., Behnke et al. 2000) that

$$
\lambda_{j, s}^{I I I, N} \leq \lambda_{j, s} \leq \lambda_{j, s}^{I I I, D}
$$

for all $j$ for which $\lambda_{j, s}<\pi^{2} / b^{2}$. Hence

$$
\frac{\pi^{2}}{a^{2}}(j-1)^{2}+\frac{\pi^{2}}{d^{2}} \leq \lambda_{j, s} \leq \frac{\pi^{2}}{a^{2}}(j-1 / 2)^{2}+\frac{\pi^{2}}{d^{2}} .
$$

For the antisymmetric problem the eigenfunction expansions and eigenvalues can be recalculated and (3.54) becomes

$$
\frac{\pi^{2}}{a^{2}}(j-1 / 2)^{2}+\frac{\pi^{2}}{d^{2}} \leq \lambda_{j, a} \leq \frac{\pi^{2}}{a^{2}} j^{2}+\frac{\pi^{2}}{d^{2}}
$$

The result of superimposing these intervals onto Figure 3 is shown in Figure 5 . Each mode appears between two estimates as required, and it can be seen that as $a / d$ increases along 


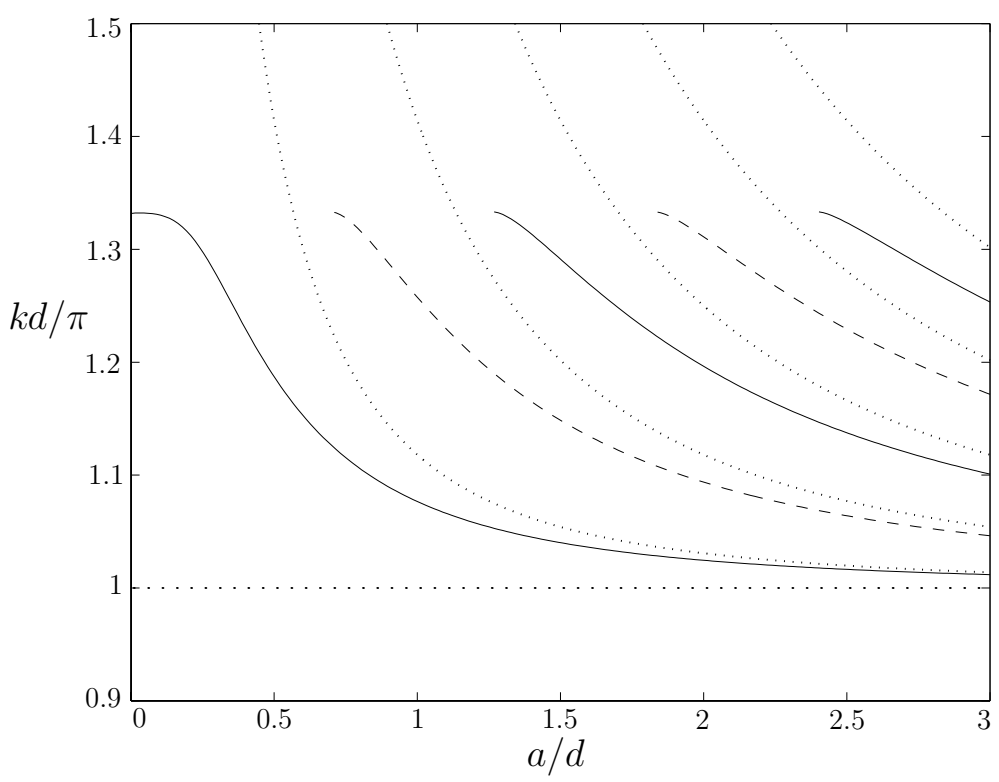

Figure 5: Bound-state energies, $k d / \pi$, for modes symmetric and antisymmetric about $x=0$ for the full solution, (solid and dashed, respectively), and the estimated intervals (dotted), plotted against $a / d$ when $b / d=0.75$.

each curve, the estimate coming from the upper bound gives a better approximation to the energy.

Each interval of (3.54) and (3.55) whose right-end value is less than $\pi^{2} / b^{2}$ contains precisely one eigenvalue. Thus there are at least $n_{s}$ symmetric bound states provided

$$
a / d>\left(n_{s}-\frac{1}{2}\right) / \sqrt{d^{2} / b^{2}-1} .
$$

Similarly, there are at least $n_{a}$ antisymmetric bound states provided

$$
a / d>n_{a} / \sqrt{d^{2} / b^{2}-1} .
$$

As $b / d \rightarrow 1$ the required value of $a / d$ tends to infinity in both cases. Similar results were found by Exner et al. (1996).

\section{Bound states below the second cut-off}

In this section we look for bound states whose energies are above the cut-off given by (2.17), i.e. $k b>\pi$. Any such mode corresponds to an eigenvalue embedded in the continuous spectrum of the relevant operator. Numerical evidence for the existence of an isolated trapped mode embedded in the continuous spectrum was given by Evans and Porter (1998) for the case of a rigid circular cylinder placed on the centre-line of a guide with either Neumann or Dirichlet boundary conditions on its walls. In each case the trapped mode was shown to occur for a single cylinder radius and at a specific frequency. 
More recently, McIver et al. (2001) showed that this trapped mode was not isolated, but part of a continuous branch of modes which exist for ellipses with varying aspect ratio. The ellipses are defined by two geometrical parameters $a$ and $b$, say, where $(x / a)^{2}+(y / b)^{2}=1$, and embedded trapped modes were found to exist for families of ellipses given by $a=a(b)$, with the corresponding frequency being of the form $k=k(b)$. Further examples of branches of embedded trapped modes in geometries defined by two geometrical parameters were computed in McIver, Linton, and Zhang (2002).

In general, it appears that for a given energy there will be a number of possible wavelike modes which can transmit the energy down the guide and in order for a bound state to exist the amplitudes of these modes must be zero. If one considers a geometry defined by a sufficient number of parameters then it may be possible to determine relationships between these parameters which lead to bound states. The numbers of parameters needed will depend on the energy band considered and the boundary conditions for the problem (see, e.g., Linton et al. 2002).

Here we will use this idea to find bound states between the first and second cut-offs for a coupled waveguide (which is defined by two geometrical parameters). We set up the problem with similar eigenfunction expansions to those in section 2. The second cut-off is defined as the energy below which only one mode can propagate in the region $x>a$, i.e. one mode in either region $I$ or region $I I$. We also restrict the energy in such a way that two wave-like modes exist in the inner region, the reason for which will become clear later. Thus we assume

$$
\begin{aligned}
& 2 \pi<k d<\frac{d \pi}{c}, \quad \text { when } 1 / 2<b / d \leq 2 / 3, \\
& 2 \pi<k d<\frac{2 d \pi}{b}, \quad \text { when } 2 / 3 \leq b / d<1 .
\end{aligned}
$$

For the symmetric problem the eigenfunction expansions for the three regions are

$$
\begin{array}{lll}
\phi_{1}(x, y) & =\sum_{n=1}^{\infty} U_{n}^{(1)} \frac{\mathrm{e}^{-\alpha_{n}(x-a)}}{-\alpha_{n}} \Psi_{n}^{(1)}(y), & \alpha_{n}=\left(\nu_{n}^{2}-k^{2}\right)^{1 / 2}, \\
\phi_{2}(x, y)=\sum_{n=2}^{\infty} U_{n}^{(2)} \frac{\mathrm{e}^{-\beta_{n}(x-a)}}{-\beta_{n}} \Psi_{n}^{(2)}(y), & \beta_{n}=\left(\mu_{n}^{2}-k^{2}\right)^{1 / 2}, \\
\phi_{3}(x, y)=\sum_{n=0}^{\infty} U_{n}^{(3)} \frac{\cosh \gamma_{n} x}{\gamma_{n} \sinh \gamma_{n} a} \Psi_{n}^{(3)}(y), & \gamma_{n}=\left(\lambda_{n}^{2}-k^{2}\right)^{1 / 2},
\end{array}
$$

where $\Psi_{n}^{(1)}(y), \Psi_{n}^{(2)}(y)$ and $\Psi_{n}^{(3)}(y)$ are given by (2.10)-(2.12). These expansions are the same as (2.14)-(2.16) except that the sum in region $I I$ starts from $n=2$, as we set the amplitude of the mode corresponding to $\beta_{1}$ equal to zero. With the restriction of frequency given by (4.1) and (4.2) $\gamma_{0}$ and $\gamma_{1}$ (and $\beta_{1}$ ) are purely imaginary whereas $\alpha_{1}$, $\alpha_{n}, \beta_{n}$ and $\gamma_{n}, n \geq 2$ are all real and positive. 
After matching on $x=a$ we obtain

$$
\sum_{n=1}^{\infty} U_{n}\left(\frac{1}{\alpha_{n}-\gamma_{m}}+\frac{\zeta_{m}}{\alpha_{n}+\gamma_{m}}\right)-\sum_{n=2}^{\infty} V_{n}\left(\frac{1}{\beta_{n}-\gamma_{m}}+\frac{\zeta_{m}}{\beta_{n}+\gamma_{m}}\right)=0, \quad m \in \mathbb{N}_{0}
$$

where $U_{n}, V_{n}$ and $\zeta_{m}$ are defined in (3.9). The only difference between (4.6) and (3.8) is that the summation for $V_{n}$ starts from $n=2$ instead of $n=1$. The condition (3.10) describing the behaviour of $U_{n}$ and $V_{n}$ for large $n$ remains the same.

We now consider the same quantities as in (3.30), except that now $f(z)$ must have simple poles at $z=\alpha_{n}, n \in \mathbb{N}$, and $z=\beta_{n}, n \geq 2$ as well as being $o(1)$ as $z \rightarrow \infty$. We choose

$$
f(z)=\exp (-z \Theta) g(z) h(z),
$$

where $\Theta$ is given by (3.24) and

$$
g(z)=\frac{1}{1-z / \alpha_{1}} \prod_{n=2}^{\infty} \frac{1-z / \gamma_{n}}{\left(1-z / \alpha_{n}\right)\left(1-z / \beta_{n}\right)}, \quad h(z)=1+\sum_{n=2}^{\infty} \frac{A_{n}}{z-\gamma_{n}} .
$$

The function $g(z)$ has the same behaviour as $z \rightarrow \infty$, as that given in (3.20) since one linear factor has been removed from both the numerator and the denominator. Cauchy's residue theorem then gives

$$
\begin{aligned}
\sum_{n=1}^{\infty} R\left(f: \alpha_{n}\right)\left(\frac{1}{\alpha_{n}-\gamma_{m}}+\frac{\zeta_{m}}{\alpha_{n}+\gamma_{m}}\right)+ & \sum_{n=2}^{\infty} R\left(f: \beta_{n}\right)\left(\frac{1}{\beta_{n}-\gamma_{m}}+\frac{\zeta_{m}}{\beta_{n}+\gamma_{m}}\right) \\
& +f\left(\gamma_{m}\right)+\zeta_{m} f\left(-\gamma_{m}\right)=0, \quad m \in \mathbb{N}_{0}
\end{aligned}
$$

and hence $U_{n}=R\left(f: \alpha_{n}\right)$ and $V_{n}=-R\left(f: \beta_{n}\right)$ provided (3.10) is satisfied and

$$
f\left(\gamma_{m}\right)+\zeta_{m} f\left(-\gamma_{m}\right)=0, \quad m \in \mathbb{N}_{0}
$$

The coefficients $A_{n}, n=2,3, \ldots$, can be found from a real, exponentially convergent system of equations.

$$
A_{m}+B_{m} \sum_{n=2}^{\infty} \frac{A_{n}}{\gamma_{m}+\gamma_{n}}=B_{m}, \quad m=2,3, \ldots
$$

where

$$
\begin{aligned}
B_{m}=2 \gamma_{m} \mathrm{e}^{2 \gamma_{m}(\Theta-a)} \frac{\left(\alpha_{1}-\gamma_{m}\right)\left(\alpha_{m}-\gamma_{m}\right)\left(\beta_{m}-\gamma_{m}\right)}{\left(\alpha_{1}+\gamma_{m}\right)\left(\alpha_{m}+\gamma_{m}\right)\left(\beta_{m}+\gamma_{m}\right)} \\
\quad \times \prod_{\substack{n=2 \\
n \neq m}}^{\infty} \frac{\left(1-\gamma_{m} / \alpha_{n}\right)\left(1-\gamma_{m} / \beta_{n}\right)\left(1+\gamma_{m} / \gamma_{n}\right)}{\left(1+\gamma_{m} / \alpha_{n}\right)\left(1+\gamma_{m} / \beta_{n}\right)\left(1-\gamma_{m} / \gamma_{n}\right)}
\end{aligned}
$$


Whereas in the non-embedded case we had one extra condition to be satisfied, we now have two; namely (4.10) with $m=0$ and $m=1$. With $\gamma_{j}=-\mathrm{i} \gamma_{j}^{\prime}$, where $\gamma_{j}^{\prime}=\left(k^{2}-\lambda_{j}^{2}\right)^{\frac{1}{2}}$, these conditions reduce to

$$
\mathrm{e}^{2 \mathrm{i} \gamma_{j}^{\prime} a}=-\frac{f\left(-\mathrm{i} \gamma_{j}^{\prime}\right)}{f\left(\mathrm{i} \gamma_{j}^{\prime}\right)}, \quad j=0,1 .
$$

For bound states to exist we must therefore have

$$
\gamma_{j}^{\prime}(a-\Theta)=\chi_{j}+\sigma_{j}+\left(n_{j}-\frac{1}{2}\right) \pi, \quad j=0,1,
$$

satisfied simultaneously, where

$$
\begin{aligned}
& \chi_{j}=-\tan ^{-1}\left(\frac{\gamma_{j}^{\prime}}{\alpha_{1}}\right)+\sum_{n=2}^{\infty}\left(\tan ^{-1}\left(\frac{\gamma_{j}^{\prime}}{\gamma_{n}}\right)-\tan ^{-1}\left(\frac{\gamma_{j}^{\prime}}{\alpha_{n}}\right)-\tan ^{-1}\left(\frac{\gamma_{j}^{\prime}}{\beta_{n}}\right)\right), \\
& \sigma_{j}=\arg \left(h\left(-\mathrm{i} \gamma_{j}^{\prime}\right)\right)=\arg \left(1-\sum_{n=2}^{\infty} \frac{A_{n}}{\gamma_{n}+\mathrm{i} \gamma_{j}^{\prime}}\right)
\end{aligned}
$$

and $n_{0}$ and $n_{1}$ are an arbitrary pair of integers.

For the case of antisymmetry about $x=0$, the conditions change to

$$
\gamma_{j}^{\prime}(a-\Theta)=\chi_{j}+\sigma_{j}^{\prime}+n_{j} \pi, \quad j=0,1,
$$

where $\sigma_{j}^{\prime}$ is the argument of $h\left(-\mathrm{i} \gamma_{j}^{\prime}\right)$ with the $A_{n}$ coefficients coming from

$$
A_{m}-B_{m} \sum_{n=2}^{\infty} \frac{A_{n}}{\gamma_{m}+\gamma_{n}}=-B_{m}, \quad m=2,3, \ldots,
$$

and $B_{m}$ given by (4.12).

\section{Results}

For the numerical results in this section, the system of equations (4.11) and (4.18) were truncated to $5 \times 5$ systems. However, in view of the fact that no modes are found for $a / d$ smaller than about 0.7 , the approximate solution in which we set $\sigma_{j}=0, j=0,1$, gives indistinguishable results.

In Figure 6 a typical set of bound-state energies, $k d / \pi$, are plotted against $a / d$ when $b / d=0.6$, for which the relevant energy range is given by (4.1) as $2<k d / \pi<2.5$. The modes symmetric about $x=0$ are represented by a cross and the modes antisymmetric about $x=0$ are shown by a circle. A detailed view of just five of these bound states is given in Figure 7, which also shows the solid lines corresponding to solutions of (4.14) and dashed lines corresponding to solutions of (4.17) for $j=0,1$, labelled with the corresponding $n_{0}$ and $n_{1}$ values. The bound-states correspond to the intersections of two lines. Modes only exist for certain values of $a / d$, but as $a / d$ increases the number of bound states present in a small $a / d$ interval increases. 


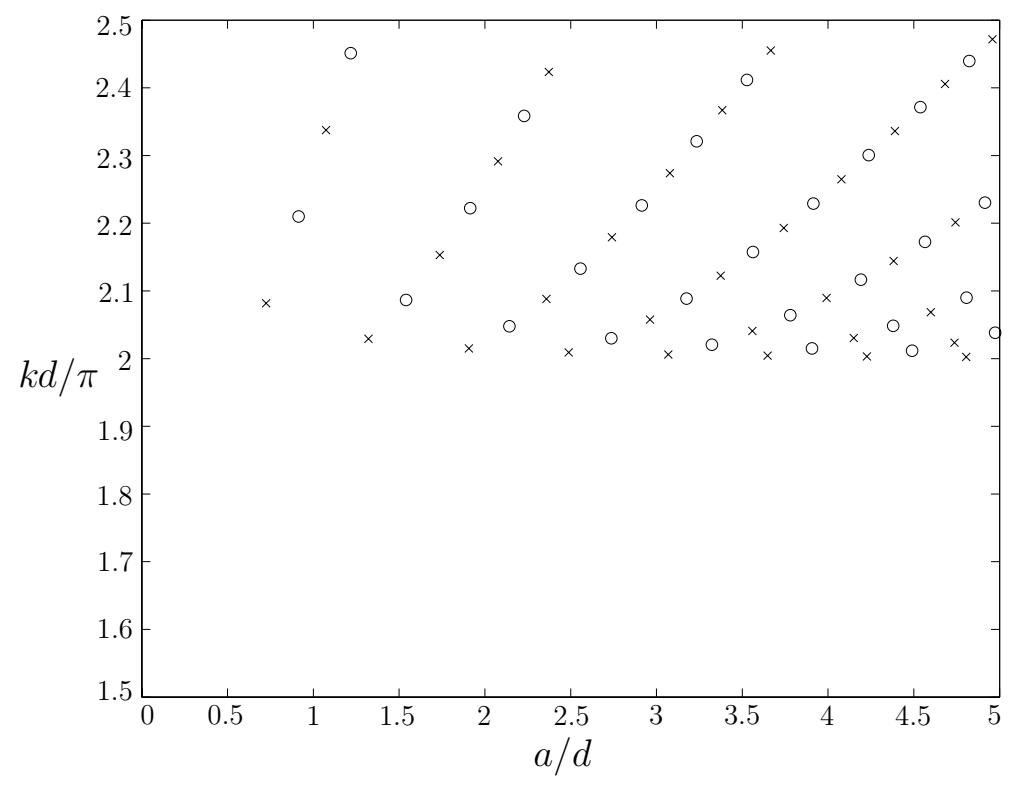

Figure 6: Bound-state energies for modes symmetric $(\times)$ and antisymmetric (o) about $x=0$ plotted against $a / d$, when $b / d=0.6$.

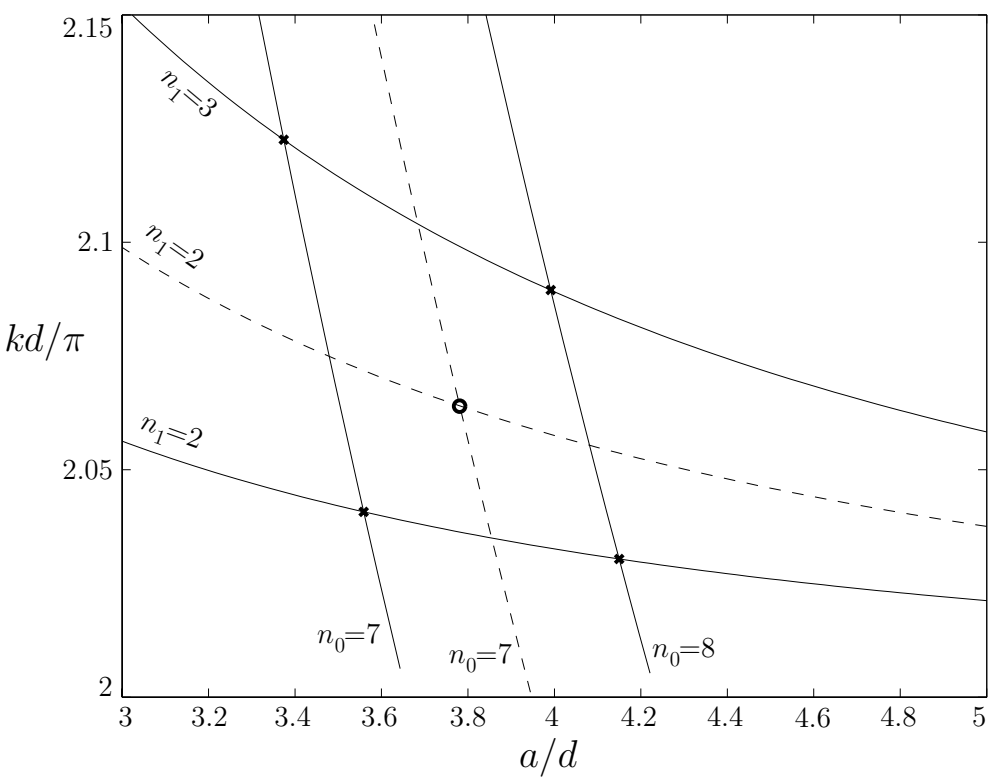

Figure 7: A detailed view of part of Figure 6 showing the curves corresponding to the solutions of (4.14) (solid curves) and (4.17) (dashed curves) for $j=0,1$, labelled with the corresponding $n_{0}$ and $n_{1}$ values. The embedded trapped modes are denoted by $\times$ (symmetric) and $\circ$ (antisymmetric). 
The bound states symmetric about $x=0$ obtained by keeping the integers $n_{0}$ and $n_{1}$ in (4.14) constant and varying either $k d / \pi$ or $a / d$ against $b / d$ are shown in Figure $8(a)$ and $(b)$, respectively. The vertical dotted line corresponds to the value $b / d=2 / 3$, and the other two dotted lines correspond to the upper cut-off's given in (4.1) and (4.2). Only a selection of results for which $a / d<2$ are given. It can be seen from Figure 8( $a)$ that the highest energy states appear when $\left(n_{0}, n_{1}\right)$ take the form $(N, N-1)$ with $N$ large and the lower energies occur when $\left(n_{0}, n_{1}\right)=(N, 1)$ with $N$ large. Figure $8(b)$ shows that the lower values of $a / d$ appear when $\left(n_{0}, n_{1}\right)$ take the form $(N, N-1)$ with $N$ small and as $n_{0}$ increases $a / d$ increases. The figures also demonstrate that modes exist for any value of $b / d$ in the range $(0.5,1)$ (but for only specific values of $a / d$ ).

In Linton et al. (2002) acoustic resonances were found between the second and third cut-off's for wave propagation down a rigid waveguide containing an off-centre rigid plate aligned with the guide walls. The coupled waveguide problem under consideration here can be set up for energies between the second and third cut-offs, with three wave-like modes appearing in the region $x<a$ and two propagating modes in the region $x>a$. Using the same techniques as above, we find that bound states exist provided three conditions are satisfied simultaneously. However, these three conditions are found to be inconsistent.

\section{The case $b / d=1 / 2$}

If $2 b=d$ the waveguide is symmetric about the mid-line parallel to the walls and this can be used to simplify the analysis. The problem can be decomposed into one symmetric about the mid-line and one antisymmetric about this line, and the latter problem is that of a parallel-plate waveguide with Dirichlet conditions on both walls, where there are no bound states. We thus restrict attention to the symmetric problem. Bound states below the first cut-off are precisely those which are obtained by letting $b / d \rightarrow 1 / 2$ in the preceding analysis.

However, in section 4 we found embedded trapped modes in the frequency range $2 \pi<k d<d \pi / c$ when $1 / 2<b / d \leq 2 / 3$. These modes do not exist when $b / d=1 / 2$. The additional symmetry in the problem when $b / d=0.5$ allows embedded modes to be found in a higher energy band in this case. In fact we now require

$$
3 \pi<k d<4 \pi
$$

to allow for two wave-like modes in the inner region and one in $x>a$. The problem is set up much as before; here we will simply present some results.

In Figure 9 the bound-state energies are plotted against $a / d$. The modes symmetric about $x=0$ are shown by crosses and the modes antisymmetric about $x=0$ by circles. Just as in Figure 7, the bound states correspond to the intersection of pairs of curves on which one of the two necessary conditions for bound states is satisfied. It can be seen that there are no bound states when $a / d$ is below some critical value and that bound states 

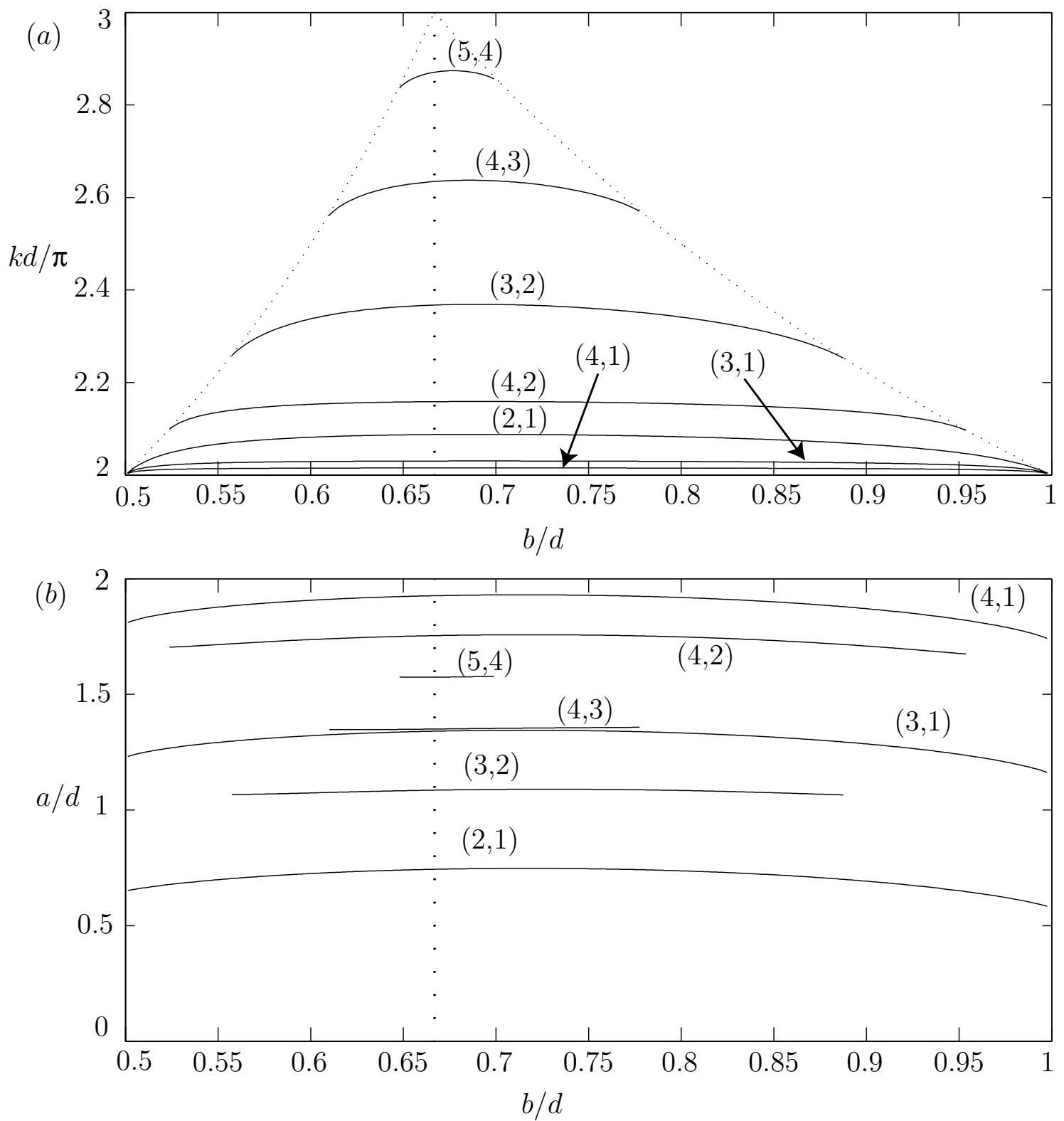

Figure 8: Variation of $(a) k d / \pi$ and $(b) a / d$ with $b / d$ for modes symmetric about $x=0$. The curves are labelled with the values of $\left(n_{0}, n_{1}\right)$ used to generate them. 


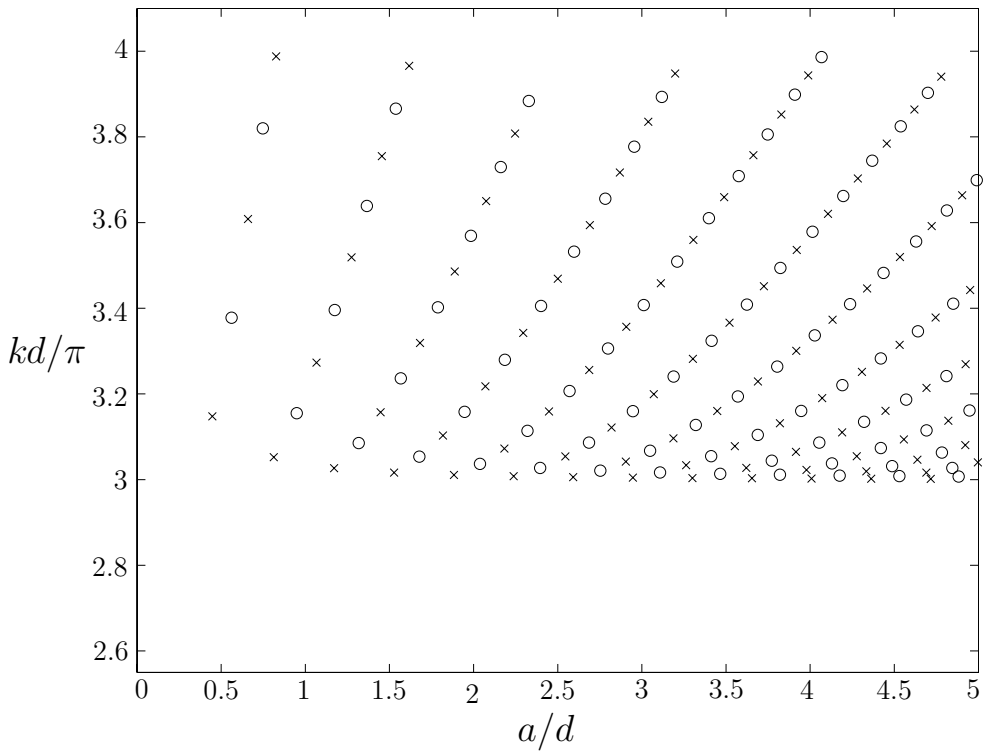

Figure 9: Bound-state energies, $k d / \pi$, for modes symmetric $(\times)$ and antisymmetric (o) about $x=0$ plotted against $a / d$ when $b / d=1 / 2$.

occur only for specific values of the plate length. As $a / d$ increases the number of trapped modes present in a small $a / d$ interval increases as before.

\section{Conclusion}

In this article we have investigated the bound states that can occur in coupled quantum wires. Specifically we have considered a two-dimensional configuration in which two parallel waveguides (of different widths) are coupled laterally through a finite length window and constructed modes which exist local to the window connecting the two guides. The geometry of the problem can be described in terms of the two parameters $a / d$ and $b / d$, where the complete guide is made up of two guides of widths $b$ and $d-b$, and the coupling window has length $2 a$.

Initially we sought modes below the first cut-off for the guide. Modes for which the energy is below this cut-off cannot propagate down the guide and so if any are found they must be localised in space. The main tool used in the analysis is the residue calculus method in which a function of a complex variable is constructed which has the property that when Cauchy's residue theorem is applied for some suitable contour, the system of equations derived from a mode-matching approach is obtained. The unknown coefficients then correspond to the residues of the complex function. If we assume that the window between the guides is long compared to the guide width then this method allows us to solve the problem explicitly, and this approximate solution can then be used as the basis for a full numerical solution. This leads to an exponentially convergent system of equations 
and high accuracy can be achieved with only a very few equations. In fact, for all but the smallest values of $a / d$, the explicit approximate solution is quite sufficient.

A number of results have been presented which show that as the window length varies bound states occur alternately symmetric and antisymmetric about the line of symmetry of the guide, and that more modes appear as the window gets larger or as the widths of the two parts of the guide get closer together (i.e. as $b / d \rightarrow 1 / 2$ ). Using a variational principle we were able to provide upper and lower bounds for the bound-state energies and prove the existence of such states for a sufficiently large window. As $a / d$ increases the bound-state energies tend towards their upper bounds.

For modes whose energies are above the first cut-off we were able to use a similar residue calculus technique to compute bound-state energies. Here we are seeking bound states whose energies are embedded in the continuous spectrum of the relevant operator and this leads to the need to satisfy two conditions simultaneously, where there was only one below the cut-off. Below the cut-off we could fix either $a / d$ or $b / d$ and vary the other to get solutions. Above the cut-off bound states occur for any values of $b / d \in(0.5,1)$ but only for specific values of the parameter $a / d$. When $b / d=1 / 2$, the region in which these embedded modes occur is shifted to a higher energy band.

\section{References}

Behnke, H., U. Mertins, M. Plum, and C. Wieners (2000). Eigenvalue inclusions via domain decomposition. Proc. Roy. Soc. Lond., A 456, 2717-2730.

Berz, F. (1951). Reflection and refraction of microwaves at a set of parallel metallic plates. Proc. IEE 98(III), 47-55.

Bulla, W., F. Gesztesy, W. Renger, and B. Simon (1997). Weakly coupled bound states in quantum waveguides. Proc. Amer. Math. Soc. 125(5), 1487-1495.

Carini, J. P., J. T. Londergan, K. Mullen, and D. P. Murdock (1992). Bound states and resonances in waveguides and quantum wires. Phys. Rev. B 46(23), 15538-15541.

Courant, R. and D. Hilbert (1953). Methods of Mathematical Physics. Volume I. Interscience Publishers, New York.

Davies, E. B. and L. Parnovski (1998). Trapped modes in acoustic waveguides. Q. J. Mech. Appl. Math. 51, 477-492.

Duclos, P. and P. Exner (1995). Curvature-induced bound states in quantum waveguides in two and three dimensions. Rev. Math. Phys. 7, 73-102.

Edmunds, D. E. and W. D. Evans (1987). Spectral Theory and Differential Operators. Clarendon Press, Oxford.

Evans, D. V. (1992). Trapped acoustic modes. IMA J. Appl. Math. 49(1), 45-60. 
Evans, D. V., M. Levitin, and D. Vassiliev (1994). Existence theorems for trapped modes. J. Fluid Mech. 261, 21-31.

Evans, D. V., C. M. Linton, and F. Ursell (1993). Trapped mode frequencies embedded in the continuous spectrum. Q. J. Mech. Appl. Math. 46(2), 253-274.

Evans, D. V. and R. Porter (1998). Trapped modes embedded in the continuous spectrum. Q. J. Mech. Appl. Math. 51(2), 263-274.

Exner, P. and P. Šeba (1989). Bound states in curved quantum waveguides. J. Math. Phys. 30(11), 2574-2580.

Exner, P., P. Šeba, M. Tater, and D. Vaněk (1996). Bound states and scattering in quantum waveguides coupled laterally through a boundary window. J. Math. Phys. 37, $4867-4887$.

Exner, P. and S. A. Vugalter (1996). Asymptotic estimates for bound states in quantum waveguides coupled laterally through a narrow window. Ann. Inst. H Poincaré: Phys. Théor. 65, 109-123.

Exner, P. and S. A. Vugalter (1997). Bound-state asymptotic estimates for windowcoupled Dirichlet strips and layers. J. Phys. A 30, 7863-7878.

Groves, M. D. (1998). Examples of embedded eigenvalues for problems in acoustic waveguides. Math. Meth. in the Appl. Sci. 21, 479-488.

Hirayama, Y., Y. Tokura, A. D. Wieck, S. Koch, R. J. Haug, K. von Klitzing, and K. Ploog (1993). Transport characteristics of a window-coupled in-plane-gated wire system. Phys. Rev. B 48(11), 7991-7998.

Hirayama, Y., A. D. Wieck, T. Bever, K. von Klitzing, and K. Ploog (1992). Parallel in-plane-gated wires coupled by a ballistic window. Phys. Rev. B 46(7), 4035-4040.

Hurd, R. A. (1954). The propagation of an electromagnetic wave along an infinite corrugated surface. Can. J. Phys. 32, 727-734.

Jones, D. S. (1953). The eigenvalues of $\nabla^{2} u+\lambda u=0$ when the boundary conditions are given on semi-infinite domains. Proc. Camb. Phil. Soc. 49, 668-684.

Jones, D. S. (1994). Methods in Electromagnetic Wave Propagation (2nd ed.). Clarendon Press, Oxford.

Khallaf, N. S. A., L. Parnovski, and D. Vassiliev (2000). Trapped modes in a waveguide with a long obstacle. Journal of Fluid Mechanics 403, 251-261.

Kunze, C. (1993). Leaky and mutually coupled wires. Phys. Rev. B 48(19), 14338-14346.

Linton, C., M. McIver, P. McIver, K. Ratcliffe, and J. Zhang (2002). Trapped modes for off-centre structures in guides. Wave Motion 36, 67-85.

Linton, C. M. and M. McIver (1998). Trapped modes in cylindrical guides. Q. J. Mech. Appl. Math. 51, 389-412. 
Martin, P. A. (1995). Asymptotic approximations for functions defined by series, with some applications in the theory of guided waves. IMA J. Appl. Math. 54, 139-157.

McIver, M., C. Linton, P. McIver, J. Zhang, and R. Porter (2001). Embedded trapped modes for obstacles in two-dimensional waveguides. Q. J. Mech. Appl. Math. 54(2), $273-293$.

McIver, M., C. Linton, and J. Zhang (2002). The branch structure of embedded trapped modes in two-dimensional waveguides. Q. J. Mech. Appl. Math. 55(2), 313-326.

Mittra, R. and S. W. Lee (1971). Analytical Techniques in the Theory of Guided Waves. Macmillan, New York.

Parker, R. (1966). Resonance effects in wake shedding from parallel plates: some experimental observations. J. Sound Vib. 4, 62-72.

Parker, R. (1967). Resonance effects in wake shedding from parallel plates: calculation of resonance frequencies. J. Sound Vib. 5, 330-343.

Parker, R. and S. A. T. Stoneman (1989). The excitation and consequences of acoustic resonances in enclosed fluid flow around solid bodies. Proc. Instn Mech. Engrs 203, 9-19.

Popov, I. Y. (2002). Asymptotics of bound states and bands for laterally coupled waveguides and layers. J. Math. Phys. 43, 215-234.

Popov, I. Y. and S. V. Frolov (2003). Three laterally coupled quantum waveguides: breaking of symmetry and resonance asymptotics. J. Phys. A 36, 1655-1670.

Takagaki, Y. and K. Ploog (1994). Ballistic electron transmission in coupled parallel waveguides. Phys. Rev. B 49(3), 1782-1788.

Whitehead, E. A. N. (1951). The theory of parallel-plate media for microwave lenses. Proc. IEE 98(III), 133-140. 